\title{
Effects of ionospheric damping on MHD wave mode structure
}

\author{
D.-H. Lee ${ }^{1}$, K.-H. Kim ${ }^{1}$, R. E. Denton ${ }^{2}$, and K. Takahashi ${ }^{3}$ \\ ${ }^{1}$ Department of Astronomy and Space Science, Kyung Hee University, Korea \\ ${ }^{2}$ Department of Physics and Astronomy, Dartmouth College, USA \\ ${ }^{3}$ The Johns Hopkins University Applied Physics Laboratory, USA
}

(Received June 19, 2004; Revised October 20, 2004; Accepted October 21, 2004)

\begin{abstract}
We calculate the mode structure of magnetospheric MHD waves on a meridional plane. We have added the effect of ionospheric dissipation to the three-dimensional dipole field MHD model of Lee and Lysak (1999); this model allows a realistic Alfven speed profile for the plasmasphere and realistic boundary conditions at the outer boundaries that vary with respect to local time. Using power spectra and plots of spatial mode structure, we show that the two-dimensional transverse modes on the dipolar meridian are strongly affected by ionospheric damping, but the compressional modes are not. The location of field line resonances spreads wide as the damping increases, but the compressional mode structure remains stable.
\end{abstract}

Key words: MHD waves, magnetosphere, ionosphere, field line resonance.

\section{Introduction}

Magnetohydrodynamic waves with wavelengths more than a few Earth radii can provide useful information about global features of the magnetosphere since the wave phenomena depend strongly on the inhomogeneity and geometry of the whole magnetosphere. Shear Alfven waves, which are called field line resonances (FLR), enable us to study the density distribution along the magnetic field lines by monitoring standing mode frequencies (Baransky et al., 1985; Waters et al., 1995; Chi and Russell, 1998; Denton et al., 2001). Compressional waves, which are strongly affected by the surrounding magnetospheric boundaries, can excite Pi 2 pulsations and/or Pc 5 waves in the magnetotail (Yeoman and Orr, 1989; Sutcliffe and Yumoto, 1991; Takahashi et al., 1995; Lee and Kim, 1999).

The magnetosphere is a highly nonuniform medium for MHD waves, and the amplitude, frequency, and polarization of ULF waves observed on the ground and in space strongly depend on the location of measurements. The ionospheric effects also play an significant role in determining the mode structure and observational feature of ULF waves. It has been studied how FLR structure and phases along the magnetic field lines are affected by the finite ionospheric conductivities (e.g., Hughes and Southwood, 1976; Newton et al., 1978; Allan and Knox, 1979; Allan, 1982; Ellis and Southwood, 1983; Budnik et al., 1998; Yoshikawa et al., 1999; Southwood and Kivelson, 2001). Therefore, it is important to investigate the details of mode structure using a realistic model. In this study, we present the two-dimensional mode structure using a three-dimensional dipole MHD model (Lee and Lysak, 1999). Effects of different values of ionospheric damping are examined by means of the power spectra and

Copy right(c) The Society of Geomagnetism and Earth, Planetary and Space Sciences (SGEPSS); The Seismological Society of Japan; The Volcanological Society of Japan; The Geodetic Society of Japan; The Japanese Society for Planetary Sciences; TERRAPUB. spatial mode structure of each MHD mode. In particular, the mode structure in this numerical experiment will be useful in understanding satellite observations of FLR and Pi2 pulsations (e.g., Osaki et al., 1998; Takahashi et al., 2003).

\section{Model}

In Lee and Lysak's dipole field MHD model (1989), the linearized MHD wave equations in a cold plasma are solved by a leapfrog scheme. It is assumed that the inner boundary is at $L=2$ and the outer boundary is at $L=10.5$, and perfect reflecting boundary conditions are used at the ionospheres, which are assumed to be at an altitude of 0.5 $\mathrm{R}_{\mathrm{E}}$. (For more details about the model, see Lee and Lysak (1999).)

Figure 1 shows the Alfven speed profile at the equator used in the model, which is based on observations (e.g., Chappell, 1988). The density along each field line is assumed to vary as $r^{-6}$, following Lee and Lysak (1989), where $r$ is the geocentric distance. This assumption enables us to obtain relatively constant Alfven speeds along each magnetic field line, which is useful for numerical convenience to avoid large computing time at the high-latitude polar region.

Ionospheric damping is a new feature of the model used in this paper. The damping coefficient $d=1-E_{\mathrm{ref}} / E_{\mathrm{in}}$, where $E_{\text {in }}$ and $E_{\text {ref }}$ represent the incident and reflected wave amplitudes, respectively. Thus $d=0$ corresponds to a perfect conducting boundary condition (no dissipation), and $d=1$ corresponds to the free boundary condition (100\% dissipation or no reflected waves). For instance, whenever the initial waves with the amplitude $E_{o}$ arrive at the ionosphere, the reflected wave amplitude would be $(1-d) E_{o}$.

Since the magnetosphere is not a perfect reflector and also asymmetric in local time, we apply different boundary conditions at the dayside and nightside outer regions. At the dayside magnetopause the Alfven speed drops from $V_{m s} \approx$ 


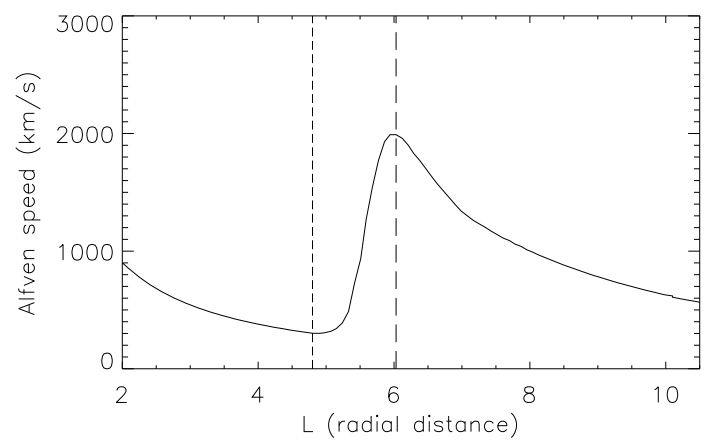

Fig. 1. The equatorial Alfven speed assumed in the model. The dashed and dash-dot curves are at $L$ values corresponding to maximum and minimum Alfven speed, respectively.

$600 \mathrm{~km} / \mathrm{s}$ in the magnetosphere to $V_{s w} \approx 60 \mathrm{~km} / \mathrm{s}$ in the solar wind (in fact, the outer region of the magnetosphere is the magnetosheath, where the Alfven speed is a little larger than that of the solar wind. Since it is still very small compared to the magnetospheric Alfven speed, we neglect such details in this model). Thus the reflection coefficient at the dayside boundary (Lee and Lysak, 1999) is given by

$$
r=\frac{V_{m s}-V_{s w}}{V_{m s}+V_{s w}}
$$

for waves propagating out of the magnetosphere. In the nightside region, compressional waves can propagate without significant speed changes through $L=10.5$ (the outer boundary of our model), so we use a free boundary there. The regions between the dayside and nightside at dawn and dusk have reflection coefficients which vary gradually from $r$ in (1) on the dayside to zero on the nightside (Lee and Lysak, 1999). Both at the ionosphere and the outer boundary in this model, the reflected waves are determined by the coefficients $d$ and $r$.

\section{Numerical Results}

We start the simulation with a radially inward propagating impulse in the magnetotail that is symmetric in latitude (thus only odd harmonic waves are excited). This impulse was used in Lee and Lysak (1999), where you find more details about the impulse. We will look at the wave spectra and spatial mode structure for different damping coefficients: no damping $(d=0.0)$, intermediate damping $(d=0.1)$, and large damping $(d=0.3)$. Figure 2 shows the wave spectra near the equator and the meridional spatial mode structure of the electric field components for $d=0$. The left and the right panels represent the spectra at noon and midnight, respectively. To avoid the antinode or node regions, spectra are calculated at grid points lying on a line that is slightly off the equatorial plane. Thus, please note that the spectral feature in Figs. 2-4 is not necessarily corresponding to the mode structure across the magnetic shells at the exact equator (see figure 6 of Budnik et al. (1998)).

The radial electric field component effectively shows the shear Alfven waves or transverse waves, while the east-west component shows the compressional waves. As confirmed in Lee and Lysak (1999) (see their figure 3 for the magnetic field spectra when $d=0.0$ is assumed), the nightside equa-
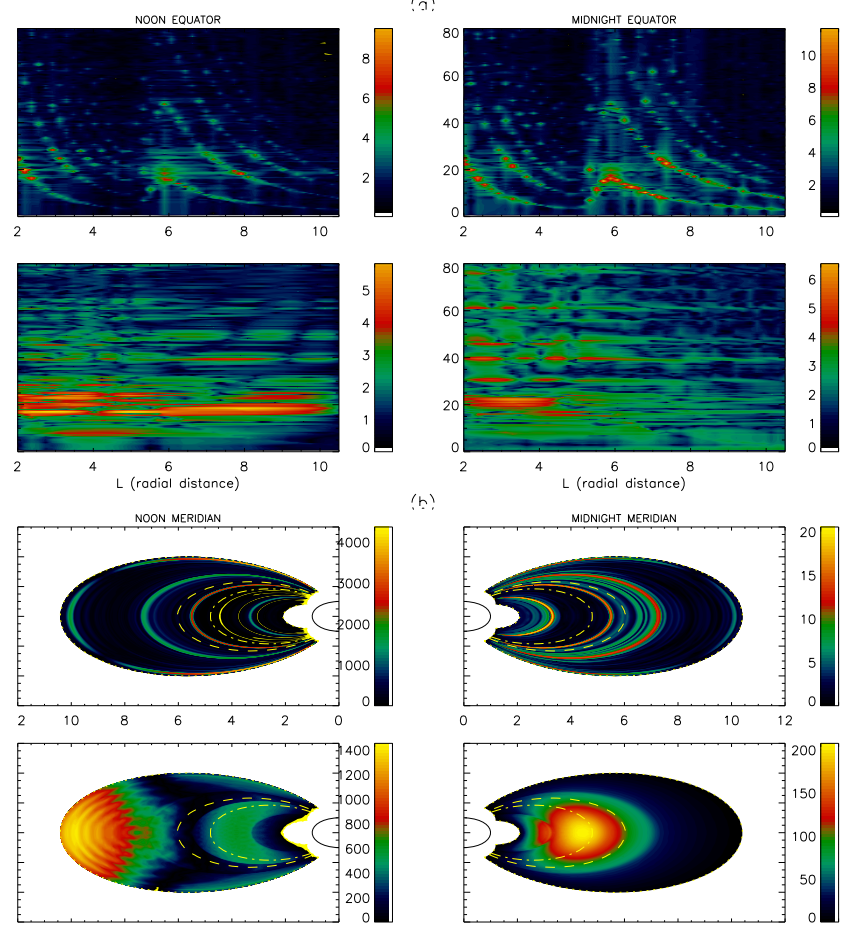

Fig. 2. No ionospheric damping is assumed $(d=0.0)$ : (a) the power spectra of the electric field components at the equator and (b) the meridional spatial mode structure for $f=8 \mathrm{mHz}$. The dashed and dash-dot curves are plotted at $L$ values corresponding to maximum and minimum Alfven speed in Fig. 1, respectively.

torial region has nearly harmonic peaks in the east-west component (compressional mode) around $f=8,16-23,30,40$ $\mathrm{mHz}$, and so on. These peaks show up most distinctly inside the plasmapause; the outer magnetosphere has less distinctive spectral features. For both power spectra corresponding to the dayside in Fig. 2(a), some of plasmaspheric modes are still found and relatively weak harmonic structure appears in the outer region. This occurs owing to the Alfven speed gradients at the dayside magnetopause. In Fig. 2(b), the meridional spatial mode structure for $f=8 \mathrm{mHz}$ is presented as an example. The mode structure is obtained by calculating the Fourier amplitude of $f=8 \mathrm{mHz}$ at each point in space during the period of $1000 \mathrm{~s}$ after the impulse. Relatively strong transverse waves that are coupled to the $f=8 \mathrm{mHz}$ compressional mode appear both in the inner and outer regions (radial component). Note that the initial impulse was assumed to be symmetric with respect to the equator, so only the fundamental mode and its odd harmonics are excited.

The nightside east-west electric field (compressional mode) in Fig. 2(b) has relatively large amplitude just inside the plasmapause where the Alfven speed becomes lowest (Lee and Kim, 1999). This is understandable considering that the electric field for this lowest frequency harmonic is likely to have largest power in the region corresponding to the minimum Alfven speed that is surrounded by "walls" with larger Alfven speed (Lee and Kim, 1999). It is interesting to note that this mode is not confined within the plasmasphere and the amplitude is rather extended beyond the plasmapause (or beyond the region of maximum Alfven speed). This fact is consistent with the properties of plasma- 

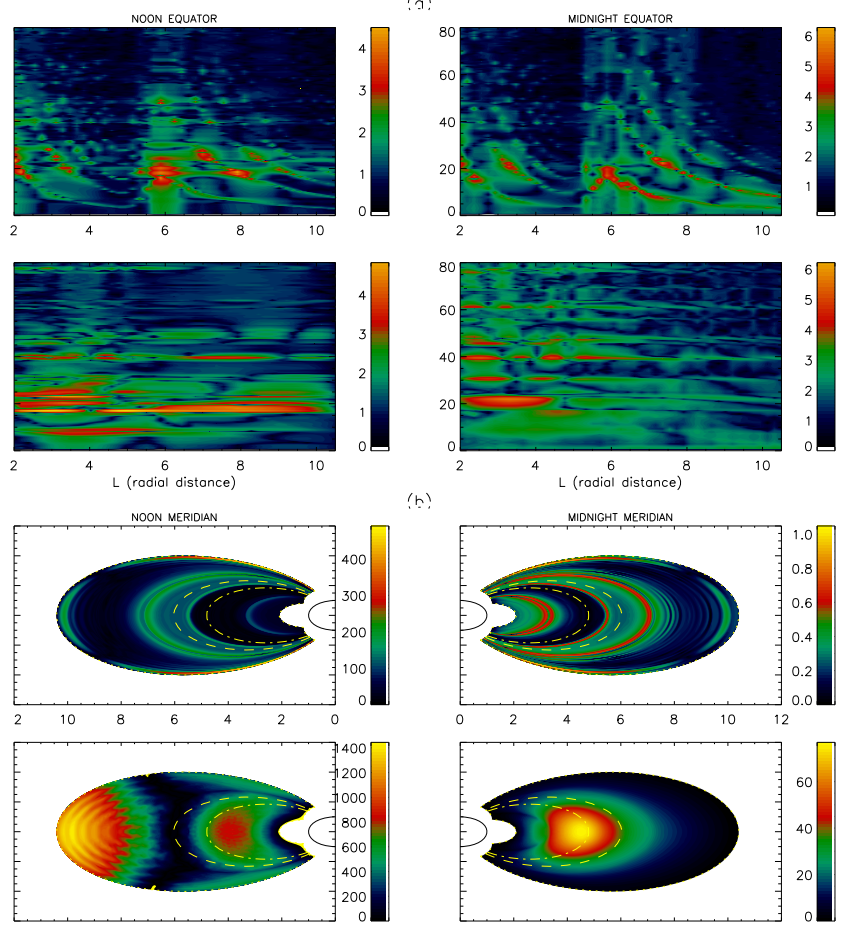

Fig. 3. Same as Fig. 2 except that intermediate ionospheric damping is assumed $(d=0.1)$.

spheric virtual resonances (Lee and Kim, 1999; Fujita et al., 2001) that arise owing to imperfect reflection at the plasmapause.

Figure 3 shows the case of $d=0.1$. In Fig. 3(a), the continuous frequency bands of the radial component (transverse mode) become relatively wide compared to Fig. 2(a). For instance, it is found that the spectrum becomes relatively broadband in the ambient region near $L=6$. However, there are no significant changes in the spectral features of the eastwest component (compressional mode). This point is consistent with the mode structure displayed in Fig. 3(b). The compressional mode structure remains almost the same as that shown in Fig. 2(b) in both global shape and amplitude. However, the transverse mode structure that was previously localized becomes spread over neighboring magnetic shells, and the amplitude significantly decreases compared to the case of no ionospheric damping.

Figure 4 shows the case of $d=0.3$. The continuous frequency bands of the radial component (transverse mode) power spectrum become even thicker, while the spectrum of the east-west component (compressional mode) seems similar to those of Figs. 2 and 3. This feature is also observed in the spatial mode structure. The east-west component (compressional mode) remains close to that in Figs. 2 and 3, including the amplitude. Therefore, it is evident from Figs. 2(b)-4(b) that the amplitude of the radial component (transverse waves) significantly decreases and the radial width of the spectral features increases as the damping coefficient increases, while the east-west component (compressional mode) amplitude is fairly stable for different damping coefficients.
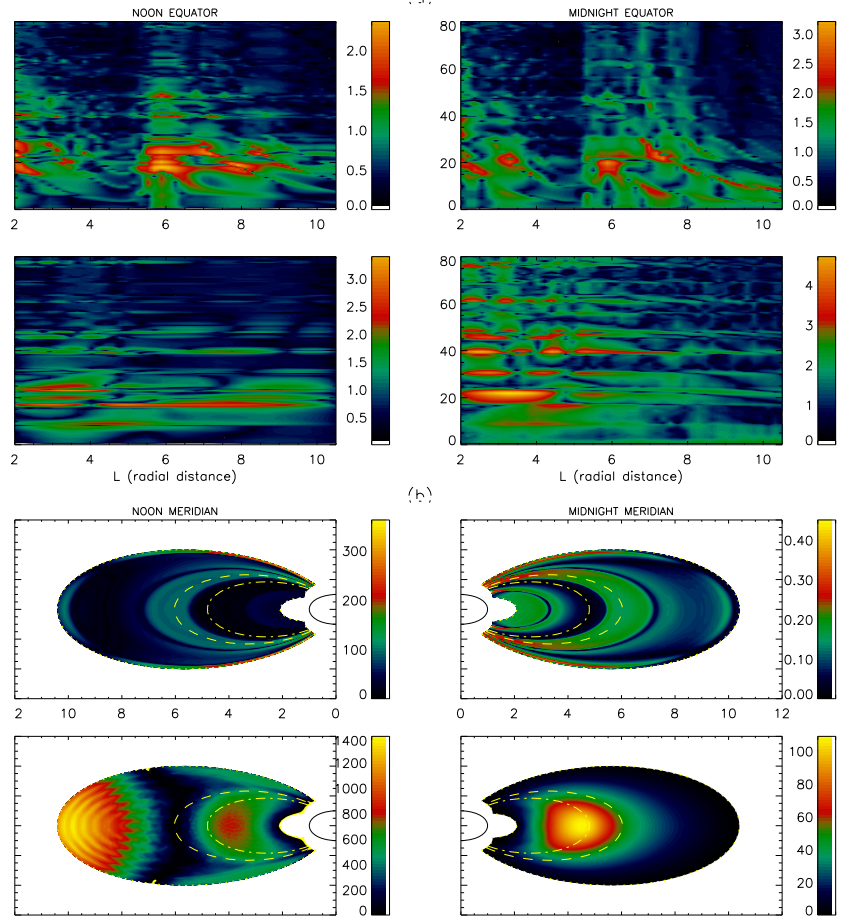

Fig. 4. Same as Fig. 2 except that strong ionospheric damping is assumed $(d=0.3)$.

\section{Discussion and Summary}

The meridional spatial mode structure presented above is limited to the case of $f=8 \mathrm{mHz}$. However, the features distinguishing transverse and compressional mode structure for $f=8 \mathrm{mHz}$ are also found at other spectral peaks, such as $f=16-23,30,40 \mathrm{mHz}$ (not shown). In both power spectrum and spatial structure, transverse modes are strongly affected by the damping rate, while the compressional modes are not. The results shown here are also indicative of the effects of other ionospheric damping coefficients, such as $d=0.8,0.5$, and so on.

In eigenmode analysis of coupled wave equations, damping is often expressed using a complex frequency $\omega=$ $\omega_{r}+i \gamma$. For finite $\gamma$, it is expected that both transverse and compressional waves will decay via dissipation. As $\gamma$ increases, the spectral peaks become more broadband while the peak frequency $\omega_{r}$ remains the same. This feature is consistent with our numerical results above in Figs. 3 and 4. It should be noted that $\gamma$ represents an average value resulting from a Fourier transform over infinite time. In a very long simulation, both transverse and compressional modes must eventually have the same damping rate. However, our studies suggest that the damping rates are very different when the damping is assumed only at the ionospheres in a finitetime simulation. This differential time-dependent behavior will be important in interpreting MHD wave properties associated with ionospheric damping.

We assumed that the incident waves at the ionosphere are reflected with the reduced amplitude for a given damping rate. In general, the reflection coefficient can be complex if we include the phase relation at the boundary (e.g., Yoshikawa et al., 1999). We neglected such phase variations at the ionosphere for simplicity in this study. In addition, 
ionospheric damping rates are assumed to be uniform in our model. In reality, the nightside damping should be more significant than the dayside damping. Effects of such nonuniform ionospheric conductivities remain as future work.

Acknowledgments. Work at Kyung Hee University was supported by the Korea Science \& Engineering Foundation grant R14-2002043-01000-0. Work at Dartmouth College was supported by NSF grant ATM-0245664.

\section{References}

Allan, W., Phase variation of ULF pulsations along a geomagnetic field line, Planet. Space Sci., 30, 339, 1982.

Allan, W. and F. B. Knox, The effects of finite ionospheric conductivities on axisymmetric toroidal Alfven waves resonances, Planet. Space Sci., 27, 939, 1979.

Baransky, L. N., J. E. Borovkov, M. B. Gokhberg, S. M. Krylov, and V. A. Troitskaya, High resolution method of direct measurement of the magnetic field lines' eigenfrequencies, Planet. Space Sci., 33, 1369, 1985.

Budnik, F., M. Stellmacher, K.-H. Glassmeier, and S. C. Buchert, Ionospheric conductance distribution and MHD wave structure: Observation and model, Ann. Geophysicae, 16, 140, 1998.

Chappell, C. R., The terrestrial plasma source: A new perspective in solarterrestrial processes from Dynamics Explorer, Rev. Geophys., 26, 229, 1988.

Chi, P. J. and C. T. Russell, An interpretation of the cross-phase spectrum of geomagnetic pulsations by the field line resonance theory, Geophys. Res. Lett., 25, 4445, 1998.

Denton, R. E., M. R. Lessard, R. Anderson, E. G. Miftakhova, and J. W. Hughes, Determining the mass density along magnetic field lines from toroidal eigenfrequencies: Polynomial expansion applied to CRRES data, J. Geophys. Res., 106, 29,915, 2001.

Ellis, P. and D. J. Southwood, Reflection of Alfven waves by nonuniform ionospheres, Planet. Space Sci., 31, 10,717, 1983.

Fujita, S., T. Mizuta, M. Itonaga, A. Yoshikawa, and H. Nakata, Propagation property of transient MHD impulses in the magnetosphere-ionosphere system: The 2D model of the Pi2 pulsation, Geophys. Res. Lett., 28, 2161, 2001 .
Hughes, J. W. and D. J. Southwood, The screening of micropulsation signals by the atmosphere and ionosphere, J. Geophys. Res., 81, 3234, 1976.

Lee, D. H. and K. Kim, Compressional MHD waves in the magnetosphere: A new approach, J. Geophys. Res., 104, 12,379, 1999.

Lee, D. H. and R. L. Lysak, Magnetospheric ULF wave coupling in the dipole model: The impulsive excitation, J. Geophys. Res., 94, 17,097, 1989.

Lee, D. H. and R. L. Lysak, MHD waves in a three-dimensional dipolar magnetic field: A search for Pi2 pulsations, J. Geophys. Res., 104, 28,691, 1999.

Newton, R. S., D. J. Southwood, and W. Hughes, Damping of geomagnetic pulsations by the ionosphere, Planet. Space Sci., 26, 201, 1978.

Osaki, H., K. Takahashi, H. Fukunishi, T. Nagatsuma, H. Oya, A. Matsuoka, and D. K. Milling, Pi2 pulsations observed from the Akebono satellite in the plasmasphere, J. Geophys. Res., 103, 17,605-17,615, 1998.

Southwood, D. J. and M. G. Kivelson, Damping standing Alfve'n waves in the magnetosphere, J. Geophys. Res., 106, 10,829, 2001.

Sutcliffe, P. R. and K. Yumoto, On the cavity mode nature of low-latitude Pi 2 pulsations, J. Geophys. Res., 96, 1543, 1991.

Takahashi, K., S.-I. Ohtani, and B. J. Anderson, Statistical analysis of Pi 2 pulsations observed by the AMPTE CCE spacecraft in the inner magnetosphere, J. Geophys. Res., 100, 21,929, 1995.

Takahashi K., D.-H. Lee, M. Nose', R. R. Anderson, and W. J. Hughes, CRRES electric field study of the radial mode structure of Pi2 pulsations, J. Geophys. Res., 108, 1210, doi:10.1029/2002JA009761, 2003.

Waters, C. L., F. W. Menk, and B. J. Fraser, Low latitude geomagnetic field line resonance: Experiment and modeling, J. Geophys. Res., 99, 17,547, 1995.

Yeoman, T. K. and D. Orr, Phase and spectral power of mid-latitude Pi 2 pulsations: Evidence for a plasmaspheric cavity resonance, Planet. Space Sci., 37, 1367, 1989.

Yoshikawa, A., M. Itonaga, S. Fujita, H. Nakata, and K. Yumoto, Eigenmode analysis of field line oscillations interacting with the ionosphereatmosphere-solid earth electromagnetic coupled system, J. Geophys. Res., 104, 28,437, 10.1029/1999JA900287, 1999.

D.-H. Lee (e-mail: dhlee@khu.ac.kr), K.-H. Kim, R. E. Denton, and K. Takahashi 